\title{
Multiple Magnetic Bilayers and Unconventional Criticality without Frustration in $\mathrm{BaCuSi}_{2} \mathrm{O}_{6}$
}

\author{
S. Allenspach $\odot,{ }^{1,2}$ A. Biffin, ${ }^{3}$ U. Stuhr, ${ }^{3}$ G. S. Tucker $\odot,{ }^{3,4}$ S. Ohira-Kawamura $\odot,{ }^{5}$ M. Kofu, ${ }^{5}$ D. J. Voneshen, ${ }^{6}$ \\ M. Boehm, ${ }^{7}$ B. Normand, ${ }^{1}$ N. Laflorencie, ${ }^{8}$ F. Mila, ${ }^{4}$ and Ch. Rüegg ${ }^{1,2}$ \\ ${ }^{1}$ Neutrons and Muons Research Division, Paul Scherrer Institut, CH-5232 Villigen, Switzerland \\ ${ }^{2}$ Department of Quantum Matter Physics, University of Geneva, CH-1211 Geneva, Switzerland \\ ${ }^{3}$ Laboratory for Neutron Scattering and Imaging, Paul Scherrer Institut, CH-5232 Villigen, Switzerland \\ ${ }^{4}$ Institute of Physics, École Polytechnique Fédérale de Lausanne (EPFL), CH-1015 Lausanne, Switzerland \\ ${ }^{5}$ J-PARC Center, Japan Atomic Energy Agency, Tokai, Ibaraki 319-1195, Japan \\ ${ }^{6}$ ISIS Facility, Rutherford Appleton Laboratory, Chilton, Didcot OX11 OQX, United Kingdom \\ ${ }^{7}$ Institut Laue Langevin, 6 Rue Jules Horowitz BP156, 38024 Grenoble Cedex 9, France \\ ${ }^{8}$ Laboratoire de Physique Théorique, CNRS and Université de Toulouse, 31062 Toulouse, France
}

(Received 4 November 2019; accepted 18 March 2020; published 30 April 2020)

\begin{abstract}
The dimerized quantum magnet $\mathrm{BaCuSi}_{2} \mathrm{O}_{6}$ was proposed as an example of "dimensional reduction" arising near the magnetic-field-induced quantum critical point (QCP) due to perfect geometrical frustration of its interbilayer interactions. We demonstrate by high-resolution neutron spectroscopy experiments that the effective intrabilayer interactions are ferromagnetic, thereby excluding frustration. We explain the apparent dimensional reduction by establishing the presence of three magnetically inequivalent bilayers, with ratios $3: 2: 1$, whose differing interaction parameters create an extra field-temperature scaling regime near the QCP with a nontrivial but nonuniversal exponent. We demonstrate by detailed quantum Monte Carlo simulations that the magnetic interaction parameters we deduce can account for all the measured properties of $\mathrm{BaCuSi}_{2} \mathrm{O}_{6}$, opening the way to a quantitative understanding of nonuniversal scaling in any modulated layered system.
\end{abstract}

DOI: 10.1103/PhysRevLett.124.177205

A foundation stone of statistical physics is the theory of classical and quantum criticality [1,2], which states that all physical properties around a quantum phase transition (QPT) obey universal scaling laws dependent only on the dimension of space $d$ and the dynamical exponent $z$ (the "dimension of time" arising from the dispersion $\omega \propto k^{z}$ of low-energy excitations). The idea that perfectly frustrated competing interactions could lead to an effective reduction of $d$ has been both proposed [3-5] and contested [6-8] to explain the physics of $\mathrm{BaCuSi}_{2} \mathrm{O}_{6}$. This $S=1 / 2$ material, known as Han purple from its use as a pigment in ancient China $[9,10]$, presents a three-dimensional (3D) stack of $\mathrm{Cu}^{2+}$ bilayers [Fig. 1(a)] with dominant antiferromagnetic (AF) dimerization, significant intrabilayer interactions, and a geometrically exact offset between adjacent bilayers, but was reported to show 2D scaling exponents around the field-induced QPT [3]. The discovery of inequivalent bilayers in $\mathrm{BaCuSi}_{2} \mathrm{O}_{6}[11,12]$ raised the question of whether frustration or structural modulation, or both, would be required to explain the apparent dimensional reduction [3]; despite intensive investigation $[4,5,7,8,12-16]$, this issue has yet to be resolved, with far-reaching implications for any layered material.

While field-driven QPTs from the "quantum disordered" dimerized state to the field-induced ordered state have remained a hot topic in quantum magnetism for multiple reasons ("Bose-Einstein condensation of magnons") [17], several recent developments make this the right time to revisit dimensional reduction in $\mathrm{BaCuSi}_{2} \mathrm{O}_{6}$. First, an $a b$ initio analysis of the magnetic interactions has suggested that the effective intrabilayer interactions are ferromagnetic (FM) [18], which would preclude a frustration scenario. Second, a systematic structural determination [16] has confirmed at minimum two inequivalent and alternating bilayer units. Third, a new generation of time-of-flight (TOF) neutron spectrometers now allows magnetic excitations in materials such as $\mathrm{BaCuSi}_{2} \mathrm{O}_{6}$ to be characterized with unprecedentedly high resolution and across multiple Brillouin zones.

In this Letter, we report the results of neutron spectroscopy experiments performed to determine the full magnetic Hamiltonian of $\mathrm{BaCuSi}_{2} \mathrm{O}_{6}$. We verify that the effective intrabilayer interaction parameter is FM, establish the presence of three inequivalent bilayers with number ratios $3: 2: 1$ and determine the very weak interbilayer interaction. We demonstrate by quantum Monte Carlo (QMC) simulations that our deduced interactions are completely consistent with all prior experimental data for the magnetization, phase diagram, layer triplet populations, and quantum critical behavior. Our conclusion that structural modulation creates 


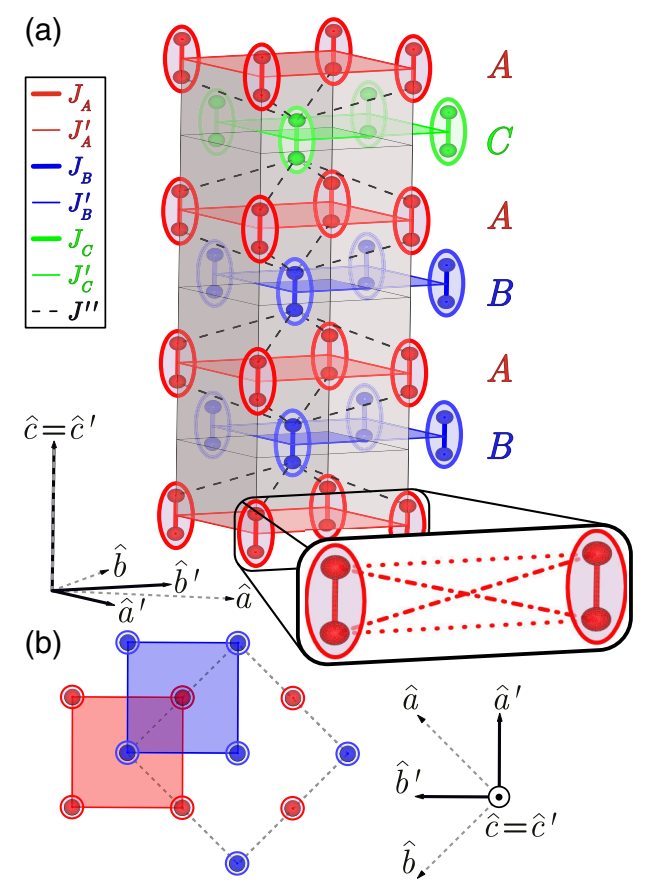

FIG. 1. (a) Schematic representation of one unit cell of the minimal magnetic model for $\mathrm{BaCuSi}_{2} \mathrm{O}_{6} ;\left\{J_{\sigma}, J_{\sigma}^{\prime}, J^{\prime \prime}\right\}$ are Heisenberg interactions. The three distinct bilayer types are labeled $A, B$, and $C$. The effective interdimer interaction parameters within each bilayer ( $J_{\sigma}^{\prime}$, edges of colored squares) result from four pairwise ionic interactions (inset). (b) Top view of the $a b$ plane. The minimal unit cell, containing one dimer per bilayer [9,22], has basis vectors $\left\{\hat{a}^{\prime}, \hat{b}^{\prime}, \hat{c}^{\prime}\right\}$; the crystallographic unit cell, used in previous scattering studies $[11,16,19]$, has basis $\{\hat{a}, \hat{b}, \hat{c}\}$ and contains two.

an additional regime of unconventional effective scaling behavior will have broad applicability in the age of designer assembly of atomically thin magnetic materials.

The room-temperature crystal structure of $\mathrm{BaCuSi}_{2} \mathrm{O}_{6}$ is tetragonal, but becomes weakly orthorhombic below $90 \mathrm{~K}$ [19-21]. As Fig. 1(a) represents, the stacked "bilayers," square lattices of $\mathrm{Cu}^{2+}$ spin dimers, have a relative shift of $\left(\begin{array}{ll}\frac{1}{2} & \frac{1}{2}\end{array}\right)$ in the $a b$ plane [in the minimal unit cell of one bilayer [9,22], represented in Fig. 1(b)]. The orthorhombic phase contains at least two structurally distinct bilayer types $[16,19]$, presumably with different intrabilayer interaction parameters, and with a very weak supercell structure showing incommensurate peaks [12] near $Q_{k}=1 / 8$ [19]. Using the crystallographic unit cell [16,19] [Fig. 1(b)] to interpret the measured dispersion relations [11] creates an ambiguity between FM and AF intrabilayer interactions, as detailed in Sec. S1 of the Supplemental Material (SM) [23], which we resolve by working in the minimal unit cell. From the experimental map of the dynamical structure factor that we obtain over multiple Brillouin zones, we demonstrate that the minimal magnetic model is that shown in Fig. 1(a) and deduce the values of all the interaction parameters.
We have performed high-resolution inelastic neutron scattering (INS) measurements on the direct-geometry TOF spectrometers AMATERAS at the J-PARC neutron source [37] and LET at ISIS [38]. Respective measurement temperatures were 0.3 and $1.6 \mathrm{~K}$. On both instruments, incident neutrons of energy $E_{i}=9 \mathrm{meV}$ observe the full band width of the magnetic excitations (3-6 meV at zero applied field). The triple-axis spectrometers TASP and EIGER at the SINQ neutron source [39] were used for further investigation of selected $\vec{Q}$ directions, measuring at $1.6 \mathrm{~K}$ on both. All experiments used one $\mathrm{BaCuSi}_{2} \mathrm{O}_{6}$ single crystal, of weight $1.01 \mathrm{~g}$, which we discuss in Sec. S2 of the SM [23].

TOF intensity data for four high-symmetry directions in $\vec{Q}$ space are shown as functions of energy transfer in Figs. 2(a)-2(d). Figures 3(a) and 3(b) show the measured mode energies and Figs. 3(c) and 3(d) their intensities, both obtained using Gaussian fits for selected $\vec{Q}$ points. The EIGER data integrate over a broad energy range to obtain high-statistics information for the combined mode intensities over an extended $|\vec{Q}|$ range. Details of data preprocessing and the Gaussian fitting procedure are presented in Sec. S3 of the SM [23].

Our results show clearly the presence of three distinct excitations in large regions of the Brillouin zone [Figs. 2(a), 2(b), 2(d), and 3(a)]. These must result from three different types of bilayer, and so we label them $A, B$, and $C$ in ascending order of energy. This confirms the result of Ref. [11], but over a much wider $|\vec{Q}|$ range. Where only two modes are visible because $B$ and $C$ are close in energy [Figs. 2(c) and 3(b)], we label the effective composite mode $B+C$.

We draw attention to three qualitative features of our data, which all lie beyond the results of Ref. [11]. (i) The minima of the strongly dispersive modes in Figs. 2(a), 2(b), and 3(a) give an unambiguous statement about the sign of the intrabilayer interactions when working in the minimal unit cell. (ii) Although the interbilayer interaction is very weak, making the bands in Fig. 2(c) almost flat, it can be determined from the variation of the intensity with $Q_{l}$. (iii) Where this band dispersion becomes $Q_{l}$ independent, in Fig. 2(d), the data can be used to establish the relative intensities of the three separate bilayer contributions.

The magnetic excitations of the dimerized $S=1 / 2$ system are "triplon" quasiparticles. To model the triplon spectrum in $\mathrm{BaCuSi}_{2} \mathrm{O}_{6}$, we generalized the method of Refs. [40,41] as outlined in Sec. S4 of the SM [23]. We assume that the only magnetic interactions are those of Fig. 1(a) and, given the weak anisotropies measured in $\mathrm{BaCuSi}_{2} \mathrm{O}_{6}$ [42], that they have purely Heisenberg character. A quantitative fit of the complete mode energy and intensity data yields the intradimer interaction parameters $J_{A}=4.275(5), J_{B}=4.72(1)$, and $J_{C}=4.95(2) \mathrm{meV}$, intrabilayer interactions $J_{A}^{\prime}=-0.480(3), J_{B}^{\prime}=-0.497(8)$, and $J_{C}^{\prime}=-0.57(1) \mathrm{meV}$, and the interbilayer interaction 

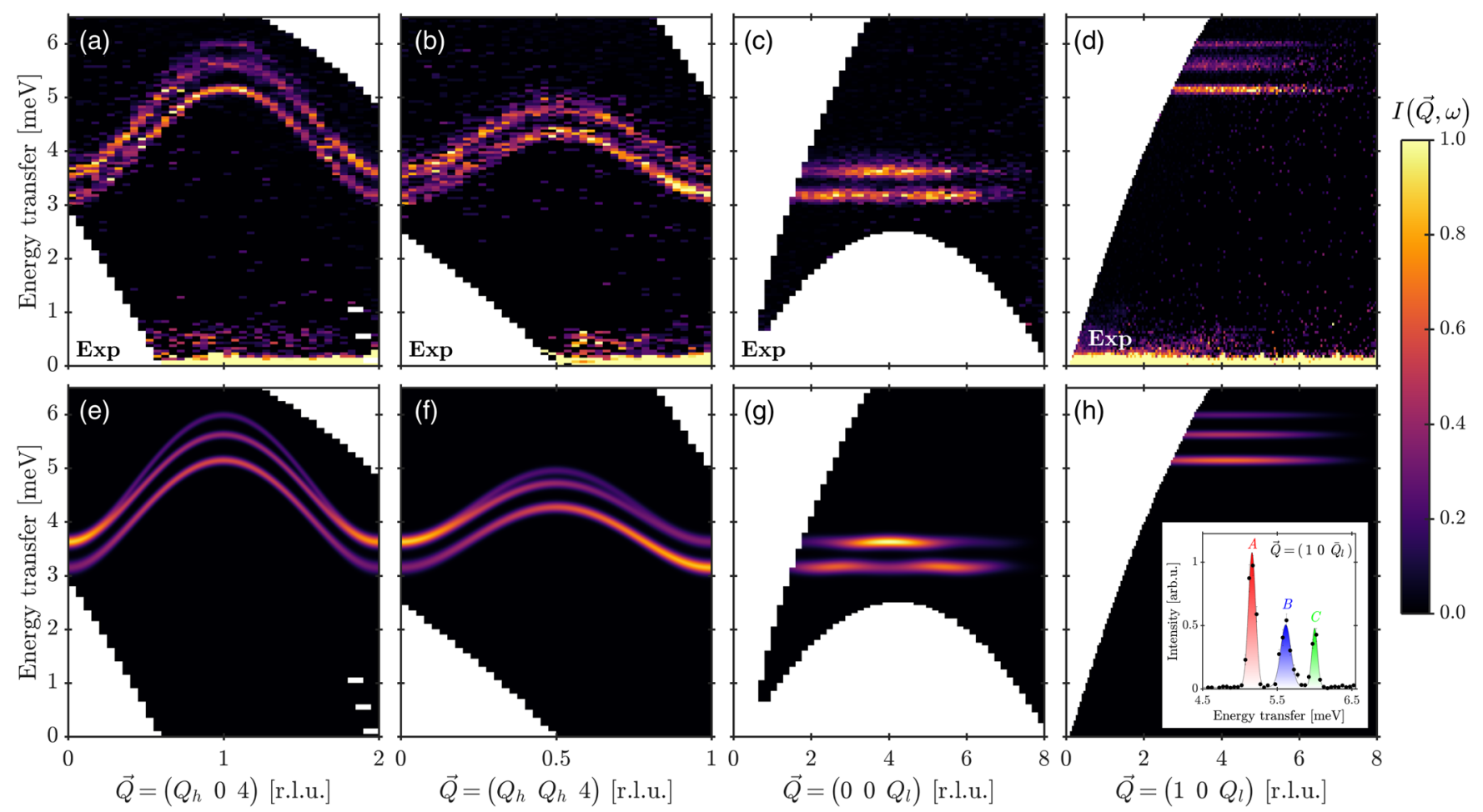

FIG. 2. (a)-(d) Intensity, $I(\vec{Q}, \omega)$, measured on AMATERAS for selected high-symmetry directions. (e)-(h) Corresponding spectra calculated with the fitted interaction parameters. $\vec{Q}$ is indexed in reciprocal lattice units (r.l.u.) of the crystallographic unit cell. Inset in (h) shows the intensity $I(\omega)$ obtained by integrating over the $\vec{Q}$ ranges $[0.95,1.05]$ in $Q_{h},[-0.05,0.05]$ in $Q_{k}$, and $[4,8]$ in $Q_{l}$ (denoted by $\bar{Q}_{l}$ ).

$J^{\prime \prime}=-0.04(1) \mathrm{meV}$, where $J>0$ refers to AF interactions and $J<0$ to FM. The quoted errors are statistical. These optimal values were used to calculate the spectra shown in Figs. 2(e)-2(h) and as the lines in Fig. 3. As part of the fitting process, we used the high- $|\vec{Q}|$ EIGER data to deduce the anisotropic magnetic form factor (Sec. S5 of the SM [23]), which confirms the concentration of spin density within the bilayers $[18,42]$. We compare our interaction parameters with those deduced from previous INS studies $[11,22]$ in Sec. S6 of the SM [23].

Returning to the primary experimental observations, (i) the positions of the minima and maxima of the dispersive modes in $\left(\begin{array}{lll}Q_{h} & 0 & 4\end{array}\right)$ and $\left(\begin{array}{lll}Q_{h} & Q_{h} & 4\end{array}\right)$ are characteristic of FM effective intrabilayer interactions. Because each $J_{\sigma}^{\prime}$ is the sum of four interionic interactions, which are generally AF, their sign indicates that the diagonal interactions represented in the inset of Fig. 1(a) are dominant [18]. For effective AF interactions, the positions of the maxima and minima would be exchanged; INS spectra calculated for intra- and interbilayer interactions of different signs are shown in Sec. S6 of the SM [23]. We stress again that FM intrabilayer interactions mean there is no interbilayer frustration in $\mathrm{BaCuSi}_{2} \mathrm{O}_{6}$.

(ii) While the very weak $J^{\prime \prime}$ results in almost flat modes along $\left(\begin{array}{llll}0 & 0 & Q_{l}\end{array}\right)$ [Figs. 2(c) and 3(b)], the mode intensities are $Q_{l}$ dependent, displaying a double-peak structure in mode $A$ with maxima at $Q_{l}=3$ and 5 [Figs. 2(c) and 3(d)]. This feature, which allows us to fit $J^{\prime \prime}$, could not be resolved at all in Ref. [11] and was revealed only by using optimized chopper settings on both high-resolution TOF spectrometers. The fact that the double peak appears in $A$ is a direct consequence of a FM $J^{\prime \prime}$; an AF interbilayer interaction would cause it to appear in $B+C$ (Sec. S6 of the SM [23]).

(iii) Because the $\left(\begin{array}{lll}1 & 0 & Q_{l}\end{array}\right)$ dispersion [Fig. 2(d)] is $Q_{l}$ invariant, it is of particular value for a quantitative determination of the relative fractions of each bilayer type. The large detector coverage of LET and AMATERAS allowed us to obtain high-quality data not available in previous experiments [11]. The $\vec{Q}$-integrated intensity shown in the inset of Fig. 2(h) establishes that the three types of bilayer are present in the approximate ratios $A: B: C=3: 2: 1$, as detailed in Sec. $\mathrm{S} 7$ of the $\mathrm{SM}$ [23]. This information, which makes completely specific the average structure reported in Ref. [16], is the foundation for the $A B A B A C$ bilayer sequence in the minimal model [Fig. 1(a)]. This model manifestly allows a highly accurate determination of the magnetic interactions and provides an excellent account of the measured spectra, in which neither the very weak orthorhombicity $[b / a=1.00167(1)$ [16] $]$ nor the incommensurability $[12,19]$ of the crystal structure plays a role.

The signs and sizes of the interaction parameters we deduce are fully consistent with the $a b$ initio analysis [18]. 


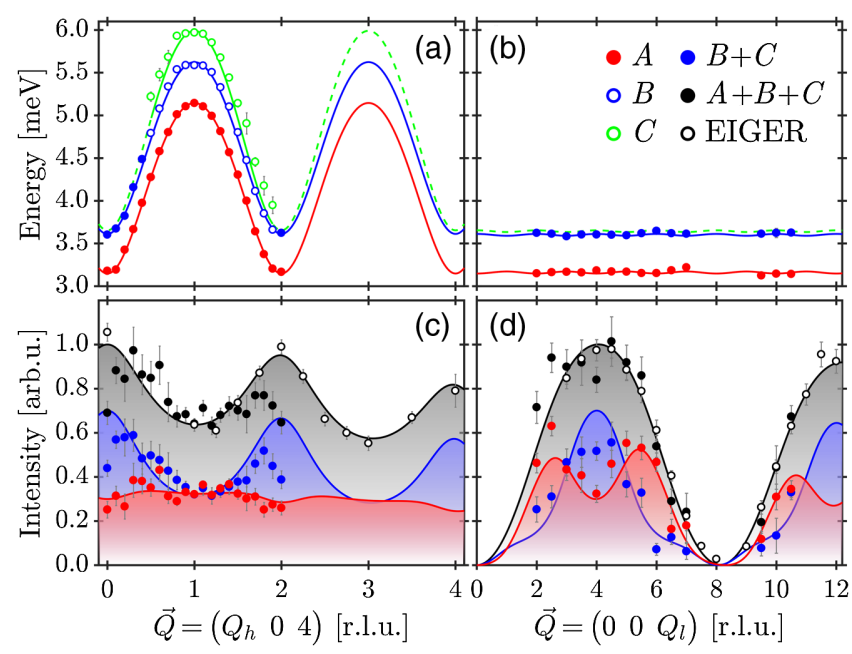

FIG. 3. Mode energies and intensities. All data other than the open circles in (c) and (d) are from AMATERAS. Lines show calculated results; in (a) and (b) the solid blue line represents $B$, whereas in (c) and (d) it represents the contribution of $B+C$. The dashed green line denotes the energy of $C$ calculated in the range where it was not distinguishable in experiment.

Assuming negligible magnetostriction, these zero-field parameters have immediate consequences for the fieldtemperature phase diagram and the field-induced QPT. Based on the initial work of Refs. [43,44], many authors have discussed their $\mathrm{BaCuSi}_{2} \mathrm{O}_{6}$ data $[3,11-13]$ and models $[4,5,7,8,14,15,45]$ by assuming $\mathrm{AF}$ intrabilayer interactions, and hence strong interbilayer frustration, begging the question of how to understand these measurements when frustration is entirely absent. To address this issue in a fully quantitative manner, we perform state-of-the-art stochastic series expansion QMC simulations [46] of the six-bilayer $(A B A B A C)$ model of Fig. 1 using the interaction parameters we determine by INS.

We simulate an effective Hamiltonian of hard-core bosons [47], to reduce the computational cost, on lattices of size $3 L^{3}$ up to $L=22$ (equivalent to 63888 spins); details are presented in Sec. S8 of the SM [23]. We calculate the temperature $T_{c}(H)$ of the field-induced ordering transition from the scaled spin stiffness $L^{(d+z-2)} \rho(L, T)$ [48], obtaining complete quantitative agreement with the phase boundary measured in Refs. [3,12,43] over the entire range of field-induced magnetic order [Fig. 4(a)]. Here we have fixed $g_{\|}=2.435$ so that the lower critical field is $H_{c 1}=23.4 \mathrm{~T}$, matching the best estimate available from nuclear magnetic resonance (NMR) [13]. Thus the agreement in Fig. 4(a) is achieved with no adjustable parameters, which, even in comparison with other well-characterized quantum magnets [17], is quite remarkable.

In Fig. 4(b) we show the field-induced triplet densities in all three bilayers at a fixed low temperature of $T=100 \mathrm{mK}$. Clearly the $A$-bilayer density $\rho_{A}$ rises rapidly and near linearly in $h=H-H_{c 1}$, whereas $\rho_{B}$ and $\rho_{C}$ rise slowly with
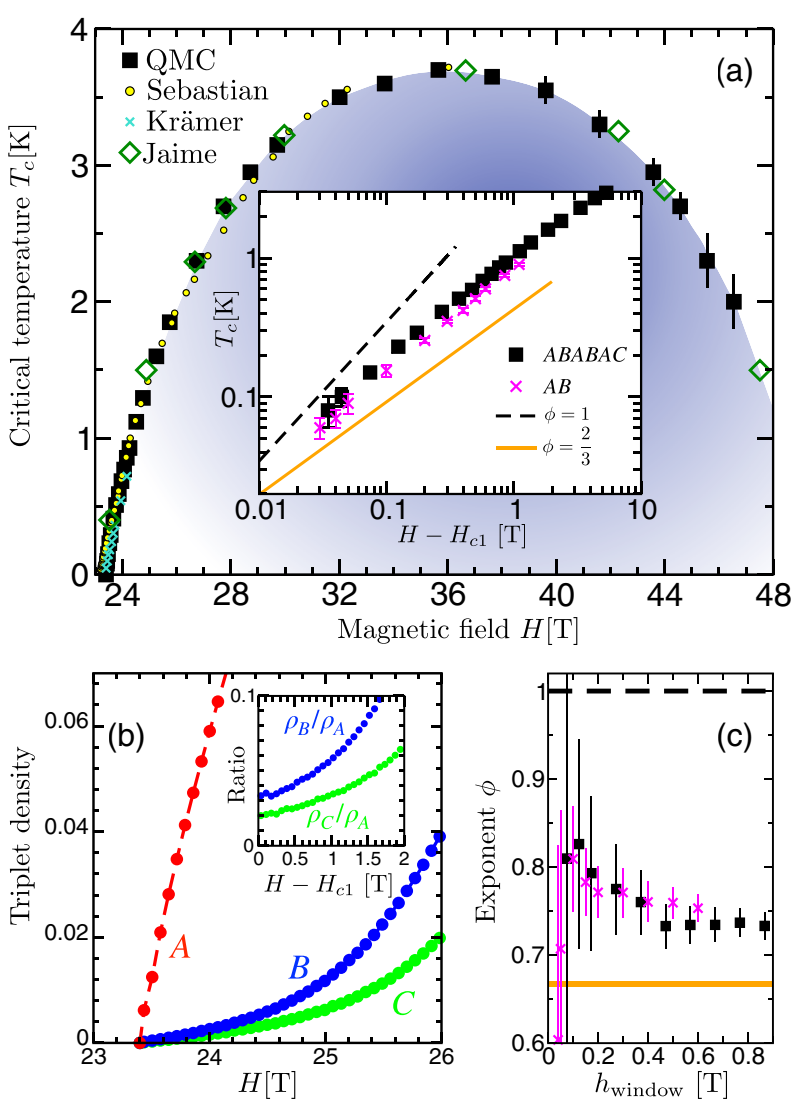

FIG. 4. QMC results for the $A B A B A C$ model. (a) $(H, T)$ phase diagram of the field-induced ordered regime, compared with experimental data from Refs. $[3,12,43]$. (Inset) Power-law scaling obtained for $A B A B A C$ and $A B$ models (see text) and compared with the scaling forms of pure $2 \mathrm{D}(\phi=1)$ and $3 \mathrm{D}(\phi=2 / 3)$ criticality. (b) Triplet populations in the three types of bilayer, computed for 1536 dimer units and shown as a function of field at $T=100 \mathrm{mK}$. (Inset) Population ratios. (c) Effective exponent $\phi$ extracted from power-law fits of the QMC data [inset (a)] to a window of width $h_{\text {window }}$.

leading linear and quadratic components, in agreement with the NMR determination of $\rho_{A}$ and $\rho_{B}$ [13]. The magnetic order parameter is determined by the effective triplet tunneling between the $A$ bilayers $t_{3 \mathrm{D}}^{\mathrm{eff}}(h)$, which is limited by the low triplet densities in the $B$ and $C$ bilayers (whose individual gaps have not yet closed). At low densities, $t_{3 \mathrm{D}}^{\text {eff }}(h)$ is proportional to the ratios $r_{B A}=\rho_{B} / \rho_{A}$ and $r_{C A}$ [14], and thus close to $H_{c 1}$ takes the generic form

$$
t_{3 \mathrm{D}}^{\mathrm{eff}}(h)=t_{c}^{\mathrm{eff}}+a_{1} h+a_{2} h^{2}+O\left(h^{3}\right)
$$

$r_{B A}$ and $r_{C A}$ grow smoothly at $H>H_{c 1}$ [inset, Fig. 4(b)] and, crucially, remain finite at $H=H_{c 1}$, giving a finite $t_{c}^{\text {eff }}$ (which is the case for any incomplete frustration $[8,13,15]$ ). Thus, as $h \rightarrow 0$, the condensed triplets have a strongly anisotropic but 3D dispersion and the critical behavior of the phase boundary is described by the exponent $\phi=z / d=$ $2 / 3$ of a fully $3 \mathrm{D}$ QPT. 
However, the structural modulation introduces a new energy scale $J_{B}-J_{A}$ and with it an energy scale $\left(J^{\prime \prime}\right)^{2} /\left(J_{B}-J_{A}\right)$ [14]. Temperatures above this latter scale (approximately $0.04 \mathrm{~K}$ ) may act to decouple the $A$ bilayers, leading to an anomalous effective exponent $\phi(h)$ over a range of $h$. This is corroborated by our QMC results for $T_{c}(H)$ in the candidate quantum critical regime [inset, Fig. 4(a)], both for the $A B A B A C$ model of $\mathrm{BaCuSi}_{2} \mathrm{O}_{6}$ and for a simplified two-layer $A B$ model giving access to lower temperatures. We extract an effective $\phi$ by power-law fits in a window of width $h_{\text {window }}$ and find [Fig. 4(c)] that it does not show accurate 3D scaling for any realistic $h_{\text {window }}$, but also never approaches 2D scaling. Only for $h_{\text {window }}<$ $0.08 \mathrm{~T}$ do our $A B$-model data suggest that the genuine $3 \mathrm{D}$ critical scaling regime is entered. Above this we demonstrate a clear crossover into an additional regime of nontrivial effective scaling, arising due to bilayer modulation, in which $\phi(h)$ is determined by the field evolution of $t_{3 \mathrm{D}}^{\text {eff }}(h)$.

We therefore verify that the $A B A B A C$ model with an unfrustrated interbilayer interaction is fully consistent with all of the previous, highly detailed experiments that have probed the properties of $\mathrm{BaCuSi}_{2} \mathrm{O}_{6}[3,11-13,16$, 19-21,42-44]. The appearance of dimensional reduction near the QPT [3] is a consequence only of the inequivalent bilayer units and not of frustration [8]. The unconventional physics in this previously unrecognized regime is contained in the effective triplet tunneling between $A$ bilayers [Eq. (1)], where $t_{c}^{\text {eff }} \neq 0$ ensures both a finite (if narrow) regime of $3 \mathrm{D}$ scaling (the true QPT is always 3D) and a nonuniversal window-dependent scaling regime with $2 / 3<\phi<1$. With rapidly improving technological capabilities for building atomically layered magnetic materials [49-54], this type of knowledge concerning emergent behavior due to layer modulation will be essential to the design of their physical properties.

In summary, we report high-resolution INS measurements over the full bandwidth of the magnetic excitations in $\mathrm{BaCuSi}_{2} \mathrm{O}_{6}$. We have determined the minimal magnetic Hamiltonian required to model the spectrum and find that it contains three different bilayer types in the ratios $3: 2: 1$. We verify that the effective intrabilayer interaction parameters are ferromagnetic, which precludes any interbilayer frustration. We perform QMC simulations of the full magnetic model to demonstrate that our parameters account with quantitative accuracy both for the entire $(H, T)$ phase boundary and for its anomalous scaling within a window of width $1 \mathrm{~K}$ near the QPT.

We thank S. Sebastian and I. Fisher for providing large, high-quality single-crystal samples and to F. Giorgianni, M. Horvatić, P. Puphal, D. Sheptyakov, R. Stern and S. Ward for helpful discussions. N. L. and F. M. are also indebted to C. Berthier and S. Krämer for an earlier collaboration on this material. This work is based in part on experiments performed at the Swiss Spallation Neutron Source SINQ at the Paul Scherrer Institute. Measurements on AMATERAS were performed based on the approved proposal No. 2015A0320. Experiments at the ISIS Pulsed Neutron and Muon Source were supported by a beam time allocation from the Science and Technology Facilities Council. We thank the Swiss National Science Foundation and the ERC Grant Hyper Quantum Criticality (HyperQC) for financial support. N.L. thanks the French National Research Agency (ANR) for support under project THERMOLOC ANR-16-CE30-0023-0. We acknowledge CALMIP (Grants No. 2018-P0677 and No. 2019-P0677) and GENCI (Grant No. 2018-A0030500225) for highperformance computing resources.

[1] J. Zinn-Justin, Quantum Field Theory and Critical Phenomena (Clarendon Press, Oxford, 2002).

[2] S. Sachdev, Quantum Phase Transitions (Cambridge University Press, Cambridge, England, 2011).

[3] S. E. Sebastian, N. Harrison, C. D. Batista, L. Balicas, M. Jaime, P. A. Sharma, N. Kawashima, and I. R. Fisher, Dimensional reduction at a quantum critical point, Nature (London) 441, 617 (2006).

[4] C. D. Batista, J. Schmalian, N. Kawashima, P. Sengupta, S. E. Sebastian, N. Harrison, M. Jaime, and I. R. Fisher, Geometric Frustration and Dimensional Reduction at a Quantum Critical Point, Phys. Rev. Lett. 98, 257201 (2007).

[5] J. Schmalian and C. D. Batista, Emergent symmetry and dimensional reduction at a quantum critical point, Phys. Rev. B 77, 094406 (2008).

[6] M. Maltseva and P. Coleman, Failure of geometric frustration to preserve a quasi-two-dimensional spin fluid, Phys. Rev. B 72, 174415 (2005).

[7] O. Rösch and M. Vojta, Quantum phase transitions and dimensional reduction in antiferromagnets with interlayer frustration, Phys. Rev. B 76, 180401(R) (2007).

[8] O. Rösch and M. Vojta, Reduced dimensionality in layered quantum dimer magnets: Frustration vs. inhomogeneous condensates, Phys. Rev. B 76, 224408 (2007).

[9] L. W. Finger, R. M. Hazen, and R. J. Hemley, $\mathrm{BaCuSi}_{2} \mathrm{O}_{6}$ : A new cyclosilicate with four-membered tetrahedral rings, Am. Mineral. 74, 952 (1989).

[10] E. W. FitzHugh and L. A. Zycherman, A purple barium copper silicate pigment from early China, Stud. Conserv. 37, 145 (1992).

[11] C. Rüegg, D. F. McMorrow, B. Normand, H. M. Rønnow, S. E. Sebastian, I. R. Fisher, C. D. Batista, S. N. Gvasaliya, C. Niedermayer, and J. Stahn, Multiple Magnon Modes and Consequences for the Bose-Einstein Condensed Phase in $\mathrm{BaCuSi}_{2} \mathrm{O}_{6}$, Phys. Rev. Lett. 98, 017202 (2007).

[12] S. Krämer, R. Stern, M. Horvatić, C. Berthier, T. Kimura, and I. R. Fisher, Nuclear magnetic resonance evidence for a strong modulation of the Bose-Einstein condensate in $\mathrm{BaCuSi}_{2} \mathrm{O}_{6}$, Phys. Rev. B 76, 100406(R) (2007).

[13] S. Krämer, N. Laflorencie, R. Stern, M. Horvatić, C. Berthier, H. Nakamura, T. Kimura, and F. Mila, Spatially resolved magnetization in the Bose-Einstein condensed 
state of $\mathrm{BaCuSi}_{2} \mathrm{O}_{6}$ : Evidence for imperfect frustration, Phys. Rev. B 87, 180405(R) (2013).

[14] N. Laflorencie and F. Mila, Theory of the Field-Induced BEC in the Frustrated Spin- $\frac{1}{2}$ Dimer Compound $\mathrm{BaCuSi}_{2} \mathrm{O}_{6}$, Phys. Rev. Lett. 102, 060602 (2009).

[15] N. Laflorencie and F. Mila, Condensate-Free Superfluid Induced by the Frustrated Proximity Effect, Phys. Rev. Lett. 107, 037203 (2011).

[16] D. V. Sheptyakov, V. Y. Pomjakushin, R. Stern, I. Heinmaa, H. Nakamura, and T. Kimura, Two types of adjacent dimer layers in the low-temperature phase of $\mathrm{BaCuSi}_{2} \mathrm{O}_{6}$, Phys. Rev. B 86, 014433 (2012).

[17] V. Zapf, M. Jaime, and C. D. Batista, Bose-Einstein condensation in quantum magnets, Rev. Mod. Phys. 86, 563 (2014).

[18] V. V. Mazurenko, M. V. Valentyuk, R. Stern, and A. A. Tsirlin, Nonfrustrated Interlayer Order and its Relevance to the Bose-Einstein Condensation of Magnons in $\mathrm{BaCuSi}_{2} \mathrm{O}_{6}$, Phys. Rev. Lett. 112, 107202 (2014).

[19] E. C. Samulon, Z. Islam, S. E. Sebastian, P. B. Brooks, M. K. McCourt, J. Ilavsky, and I. R. Fisher, Low-temperature structural phase transition and incommensurate lattice modulation in the spin-gap compound $\mathrm{BaCuSi}_{2} \mathrm{O}_{6}$, Phys. Rev. B 73, 100407(R) (2006).

[20] K. Röwer, M. Merz, G. Roth, R. Stern, R. Cerny, and T. Kimura, Description of $\mathrm{Ba}_{1+x} \mathrm{Ni}_{x} \mathrm{Rh}_{1-x} \mathrm{O}_{3}$ with $x=$ $0.1170(5)$ in superspace: modulated composite versus modulated-layer structure, Acta Crystallogr. Sect. A 62, 197 (2006).

[21] R. Stern, I. Heinmaa, E. Joon, A. A. Tsirlin, H. Nakamura, and T. Kimura, Low-temperature high-resolution solid-state (cryoMAS) NMR of han purple $\mathrm{BaCuSi}_{2} \mathrm{O}_{6}$, Appl. Magn. Reson. 45, 1253 (2014).

[22] Y. Sasago, K. Uchinokura, A. Zheludev, and G. Shirane, Temperature-dependent spin gap and singlet ground state in $\mathrm{BaCuSi}_{2} \mathrm{O}_{6}$, Phys. Rev. B 55, 8357 (1997).

[23] See Supplemental Material at http://link.aps.org/ supplemental/10.1103/PhysRevLett.124.177205, which includes Refs. [24-36], for details of the sample, unit-cell definitions, data modelling, the magnetic form factor, the relative bilayer fractions, and the quantum Monte Carlo simulations.

[24] O. Arnold et al., Mantid-Data analysis and visualization package for neutron scattering and $\mu \mathrm{SR}$ experiments, Nucl. Instrum. Methods Phys. Res., Sect. A 764, 156 (2014).

[25] Y. Inamura, T. Nakatani, J. Suzuki, and T. Otomo, Development status of software 'Utsusemi' for Chopper Spectrometers at MLF, J-PARC, J. Phys. Soc. Jpn. 82, SA031 (2013).

[26] R. A. Ewings, A. Buts, M. D. Le, J. van Duijn, I. Bustinduy, and T. G. Perring, HoRACE: Software for the analysis of data from single crystal spectroscopy experiments at time-offlight neutron instruments, Nucl. Instrum. Methods Phys. Res., Sect. A 834, 132 (2016).

[27] S. B. Haley and P. Erdös, Standard-basis operator method in the Green's-function technique of many-body systems with an application to ferromagnetism, Phys. Rev. B 5, 1106 (1972).

[28] B. Normand and C. Rüegg, Complete bond-operator theory of the two-chain spin ladder, Phys. Rev. B 83, 054415 (2011).
[29] M. Matsumoto, B. Normand, T. M. Rice, and M. Sigrist, Field- and pressure-induced magnetic quantum phase transitions in $\mathrm{TlCuCl}_{3}$, Phys. Rev. B 69, 054423 (2004).

[30] A. Furrer, J. Mesot, and T. Strässle, Neutron Scattering in Condensed Matter Physics (World Scientific, Singapore, 2009).

[31] E. Prince, International Tables for Crystallography. Volume C: Mathematical, Physical and Chemical Tables (Kluwer Academic Publishers, Dordrecht, 2004), Vol. C.

[32] R. Coldea, S. M. Hayden, G. Aeppli, T. G. Perring, C. D. Frost, T. E. Mason, S.-W. Cheong, and Z. Fisk, Spin Waves and Electronic Interactions in $\mathrm{La}_{2} \mathrm{CuO}_{4}$, Phys. Rev. Lett. 86, 5377 (2001).

[33] N. S. Headings, S. M. Hayden, R. Coldea, and T. G. Perring, Anomalous High-Energy Spin Excitations in the High- $T_{c}$ Superconductor-Parent Antiferromagnet $\mathrm{La}_{2} \mathrm{CuO}_{4}$, Phys. Rev. Lett. 105, 247001 (2010).

[34] I. A. Zaliznyak, H. Woo, T. G. Perring, C. L. Broholm, C. D. Frost, and H. Takagi, Spinons in the Strongly Correlated Copper Oxide Chains in $\mathrm{SrCuO}_{2}$, Phys. Rev. Lett. 93, 087202 (2004).

[35] A. C. Walters, T. G. Perring, J.-S. Caux, A. T. Savici, G. D. Gu, C.-C. Lee, W. Ku, and I. A. Zaliznyak, Effect of covalent bonding on magnetism and the missing neutron intensity in copper oxide compounds, Nat. Phys. 5, 867 (2009).

[36] M. Campostrini, A. Pelissetto, P. Rossi, and E. Vicari, Determination of the critical exponents for the $\lambda$ transition of ${ }^{4} \mathrm{He}$ by high-temperature expansion, Phys. Rev. B 61, 5905 (2000).

[37] K. Nakajima, S. Ohira-Kawamura, T. Kikuchi, M. Nakamura, R. Kajimoto, Y. Inamura, N. Takahashi, K. Aizawa, K. Suzuya, K. Shibata, T. Nakatani, K. Soyama, R. Maruyama, H. Tanaka, W. Kambara, T. Iwahashi, Y. Itoh, T. Osakabe, S. Wakimoto, and M. Arai, AMATERAS: A cold-neutron disk chopper spectrometer, J. Phys. Soc. Jpn. 80, SB028 (2011).

[38] R. I. Bewley, J. W. Taylor, and S. M. Bennington, LET, a cold neutron multi-disk chopper spectrometer at ISIS, Nucl. Instrum. Methods Phys. Res., Sect. A 637, 128 (2011).

[39] U. Stuhr, B. Roessli, S. Gvasaliya, H. M. Rønnow, U. Filges, D. Graf, A. Bollhalder, D. Hohl, R. Bürge, M. Schild, L. Holitzner, C. Kägi, P. Keller, and T. Mühlebach, The thermal triple-axis-spectrometer EIGER at the continuous spallation source SINQ, Nucl. Instrum. Methods Phys. Res., Sect. A 853, 16 (2017).

[40] B. Leuenberger, H.-U. Güdel, R. Feile, and J. K. Kjems, Collective excitations in the singlet-ground-state dimer system $\mathrm{Cs}_{3} \mathrm{Cr}_{2} \mathrm{Br}_{9}$, Phys. Rev. B 28, 5368 (1983).

[41] B. Leuenberger, A. Stebler, H.-U. Güdel, A. Furrer, R. Feile, and J. K. Kjems, Spin dynamics of an isotropic singletground-state antiferromagnet with alternating strong and weak interactions: An inelastic-neutron-scattering study of the dimer compound $\mathrm{Cs}_{3} \mathrm{Cr}_{2} \mathrm{Br}_{9}$, Phys. Rev. B 30, 6300 (1984).

[42] S. Zvyagin, J. Wosnitza, J. Krzystek, R. Stern, M. Jaime, Y. Sasago, and K. Uchinokura, Spin-triplet excitons in the $S=\frac{1}{2}$ gapped antiferromagnet $\mathrm{BaCuSi}_{2} \mathrm{O}_{6}$ : Electron paramagnetic resonance studies, Phys. Rev. B 73, 094446 (2006).

[43] M. Jaime, V. F. Correa, N. Harrison, C. D. Batista, N. Kawashima, Y. Kazuma, G. A. Jorge, R. Stern, I. Heinmaa, S. A. Zvyagin, Y. Sasago, and K. Uchinokura, Magnetic-Field-Induced Condensation of Triplons in Han 
Purple Pigment $\mathrm{BaCuSi}_{2} \mathrm{O}_{6}$, Phys. Rev. Lett. 93, 087203 (2004).

[44] S. E. Sebastian, P. A. Sharma, M. Jaime, N. Harrison, V. Correa, L. Balicas, N. Kawashima, C. D. Batista, and I. R. Fisher, Characteristic Bose-Einstein condensation scaling close to a quantum critical point in $\mathrm{BaCuSi}_{2} \mathrm{O}_{6}$, Phys. Rev. B 72, 100404(R) (2005).

[45] Y. Kamiya, N. Kawashima, and C. D. Batista, Finitetemperature transition in the spin-dimer antiferromagnet $\mathrm{BaCuSi}_{2} \mathrm{O}_{6}$, J. Phys. Soc. Jpn. 78, 094008 (2009).

[46] O. F. Syljuåsen and A. W. Sandvik, Quantum Monte Carlo with directed loops, Phys. Rev. E 66, 046701 (2002).

[47] F. Mila, Ladders in a magnetic field: A strong coupling approach, Eur. Phys. J. B 6, 201 (1998).

[48] A.W. Sandvik, Critical Temperature and the Transition from Quantum to Classical Order Parameter Fluctuations in the Three-Dimensional Heisenberg Antiferromagnet, Phys. Rev. Lett. 80, 5196 (1998).

[49] M. Gibertini, M. Koperski, A. F. Morpurgo, and K. S. Novoselov, Magnetic 2D materials and heterostructures, Nat. Nanotechnol. 14, 408 (2019).

[50] D. R. Klein, D. MacNeill, Q. Song, D. T. Larson, S. Fang, M. Xu, R. A. Ribeiro, P. C. Canfield, E. Kaxiras, R. Comin, and P. Jarillo-Herrero, Enhancement of interlayer exchange in an ultrathin two-dimensional magnet, Nat. Phys. 15, 1255 (2019).

[51] N. Ubrig, Z. Wang, J. Teyssier, T. Taniguchi, K. Watanabe, E. Giannini, A.F. Morpurgo, and M. Gibertini, Lowtemperature monoclinic layer stacking in atomically thin $\mathrm{CrI}_{3}$ crystals, 2D Mater. 7, 015007 (2019).

[52] X. Cai, T. Song, N. P. Wilson, G. Clark, M. He, X. Zhang, T. Taniguchi, K. Watanabe, W. Yao, D. Xiao, M. A. McGuire, D. H. Cobden, and X. Xu, Atomically Thin $\mathrm{CrCl}_{3}$ : An InPlane Layered Antiferromagnetic Insulator, Nano Lett. 19, 3993 (2019).

[53] Z. Wang, M. Gibertini, D. Dumcenco, T. Taniguchi, K. Watanabe, E. Giannini, and A. F. Morpurgo, Determining the phase diagram of atomically thin layered antiferromagnet $\mathrm{CrCl}_{3}$, Nat. Nanotechnol. 14, 1116 (2019).

[54] H. H. Kim, B. Yang, S. Li, S. Jiang, C. Jin, Z. Tao, G. Nichols, F. Sfigakis, S. Zhong, C. Li, S. Tian, D. G. Cory, G.-X. Miao, J. Shan, K. F. Mak, H. Lei, K. Sun, L. Zhao, and A.W. Tsen, Evolution of interlayer and intralayer magnetism in three atomically thin chromium trihalides, Proc. Natl. Acad. Sci. U.S.A. 116, 11131 (2019). 